\title{
The Effects of Different Endoglycosidases on Erythrocyte CD44, CD47 and CD147 Purified by Affinity Chromatography
}

\author{
Mohrah Alashaikh \\ Clinical Laboratory Sciences, College of Applied Medical Sciences, King Saud University, \\ Riyadh, Kingdom of Saudi Arabia.
}

\begin{abstract}
Endoglycosidases are enzymes with the ability to cleave certain $\mathrm{N}$-and $\mathrm{O}$ - linked glycans attached to glycoproteins. The biochemical structure of some erythrocyte glycoproteins attached carbohydrate chains are not elucidate yet. The aim of this study is to investigate the effects of different endoglycosidases on three purified erythrocyte glycoproteins, CD147, CD47 and CD44, which will also provide clues about the structure of their N-linked glycans. Peptide-N-glycosidase F (PNGaseF), Endo- $\beta$-N-acetylglucosaminidase F1 (EndoF1), endo- $\beta$ $\mathrm{N}$-acetylglucosaminidase $\mathrm{H}($ EndoH) or Endo- $\beta$-galactosidase $($ Endo $\beta)$ was used to digest the glycoproteins purified by antibody high-affinity chromatography, and the results were analysed by sodium dodecyl sulfate polyacrylamide gel electrophoresis. CD147, CD47 and CD44 were purified effectively by BRIC235, BRIC126 and MA103, respectively. PNGaseF caused a significant variation in the electrophoretic mobility of the glycoproteins, while no remarkable effects of Endo $\beta$ were detected. EndoH caused a reduction in the molecular weight of CD47 and CD44, although it did not appear to cleave all the glycans. In contrast, EndoH cleaved almost all glycans from CD147. Our results indicate that most of the N-linked glycans of CD147 and CD47 are high-mannose and hybrid types. The findings suggest that a significant amount of CD44 N-glycans are from complex oligosaccharides.
\end{abstract}

Keywords: CD44; CD47; CD147; endoglycosidase; N-glycan, erythrocyte.

\section{Introduction}

Glycosylation is the most common post-translation protein modification, which indicates its importance in protein structure and function. Although not all roles of glycosylation and their effects on biological functions of proteins have been elucidated, glycosylation has been found to contribute to a number of biological functions such as protein stability, folding, the cell surface expression of several proteins, and protection against proteolytic degradation [1-3]. The main types of oligosaccharide moieties attached to proteins are $\mathrm{N}$ - and O-linked glycans. Around $90 \%$ of well-characterized glycoproteins carry the N-linked carbohydrate structure alone or with O-glycan [4].

*Corresponding Author :malalshaikh@ksu.edu.sa 
The erythrocyte plasma membrane is a unique structure with diverse antigenic and functional characteristics. The surface of erythrocytes is dominated by integral proteins and glycoproteins, whose molecular mass may comprise up to $85 \%$ carbohydrates [5]. Carbohydrates undergo some modifications associated with abnormal erythropoiesis, [6] and there is a loss in carbohydrate content as red blood cells (RBC) age [7]. Glycosylation also plays a role in the antigenic diversity of $\mathrm{RBC}$, as some antigens are carbohydrate in nature.

Endoglycosidase enzymes have been used widely to study the biostructure of glycoproteins, investigate the composition of oligosaccharide chains and examine antigenic determinants on glycoproteins or glycolipids. They have also been used to estimate the number of N-glycans on glycoproteins [8]. These enzymes have the ability to cleave glyosidic bonds at different positions, releasing oligosaccharides partly or entirely from glycoproteins and glycolipids. PNGaseF, for example, removes intact N-linked glycans from glycoproteins by cleaving the $\beta$-aspartyl-glucosamine bond between the innermost GlcNAc and Asn residues of glycosylated proteins [9]. Endo $\beta$ removes poly-N-acetyllactosamine structures from glycans. It cleaves the $\beta-(1,4)$ galactose linkage in GlcNAc $\beta-(1,3)-G a l \beta-(1,4)$ structures $[10]$.

Effects of endoglycosidase have been studied on intact and purified erythrocyte glycoproteins. Using Endo- $\beta$-N-acetylglucosaminidase $\mathrm{F}$ preparation or Endo $\beta$ on intact erythrocyte membranes demonstrates that the $\mathrm{Rh}$ molecule is not glycosylated, while cluster of differentiation (CD) 242 carries N-glycans [11]. Also, Tanner et al.[12] showed that treating intact erythrocytes with these two enzymes, alone or in combination, has various effects on N-glycans of the major erythrocyte glycoproteins. The study found that EndoF alone effectively cleaves glycans on Duffy, and the combined enzymes can cleave Band $3 \mathrm{~N}$ glycans efficiently. Also it has been shown that purified glucose transporter glycoprotein is susceptible to EndoF [13,14].

Research on glycosylation of erythrocyte glycoproteins has not yet elucidated the biochemical nature of most of these carbohydrates. This study aimed to investigate the effects of different deglycosylated enzymes on purified erythrocyte glycoproteins CD44, CD47 and CD147, by detecting the change in migration on sodium dodecyl sulfate (SDS) gels, giving insight into the glycosylation characteristics of these molecules. The present study examined the effects of a number of endoglycosidases (PNGaseF, EndoF1, EndoH and Endo $\beta$ ), alone and in combination, on these purified erythrocyte glycoproteins, interpreting them together in a single work. The different effects of these enzymes give clues about the glycoproteins' types of N-linked glycans.

\section{Materials and Methods}

All chemicals and enzymes were obtained from Sigma (Poole, Dorset, UK), unless otherwise stated. 


\section{Ethics statement}

Ethical approval was not required as the blood samples used were from anonymous donors that were provided with written informed consent for research use, in accordance with both the Declaration of Helsinki and the policy of the National Health Service Blood and Transplant.

\section{Antibodies}

\section{For protein purification.}

The monoclonal antibodies (mAbs) BRIC235 (IgG2b) to CD44 and BRIC126 to CD47 were obtained and purified from the International Blood Group Reference Laboratory (IBGRL) Research Products, Bristol, UK. CD147 clone MA103 (ascetic fluid) (gift from Dr. J. Mattes, Bristol, UK) was purified by adsorption to and elution from Protein A sepharose (PAS) beads (GE healthcare, Buckinghamshire, UK).

\section{For analysing on SDS-PAGE.}

KZ-1 (IgG1) and BRIC222 (IgG1) (IBGRL Research Products, Bristol, UK) were used for CD44. Rabbit antibodies Out-1 (in-house) was used to CD47. For CD147, biotinylated antiCD147 (R\&D system, Abingdon, UK) was used. Anti-mouse (IgG1) and anti-human (IgG) (Jackson ImmunoResearch Laboratories, West Grove, USA) were used as secondary antibodies for western blot analysis.

\section{Proteins purification}

\section{Crosslinking $m A$ bs to PAS}

The method for antibody coupling to PAS was adapted from Moser et al. [15]. BRIC235, BRIC126 and MA103 (mAb to CD44, CD47 and CD147, respectively) were concentrated in phosphate buffer saline (PBS), pH7.4. PAS (GE healthcare) slurry was washed three times in nine volumes per-wash of either PBS or high salt buffer and then incubated overnight at $4^{\circ} \mathrm{C}$ with mixing. They were centrifuged for five minutes at $196 \mathrm{~g}$ and $4^{\circ} \mathrm{C}$. Then, the antibodies were added to the beads and three volumes of fresh coupling buffers were also added. They were incubated at $4^{\circ} \mathrm{C}$ overnight with mixing. The beads were pelleted as above, and the supernatants were removed. A sample was reserved from each tube to estimate the amount of Abs left pre-coupling by measuring the optical density (OD) of the supernatant as described below. The beads then were washed two times in 10 volumes of borate buffer $\left(0.15 \mathrm{M} \mathrm{NaB}_{4}\right.$, $\mathrm{pH}$ 9.0). The beads were incubated in 10 volumes of the buffer with $20 \mathrm{mM}$ dimethyl pimelimidate (DMP) for one hour at room temperature $(\mathrm{R} / \mathrm{T})$. Beads were centrifuged, supernatants were aspirated and were then washed once with $0.2 \mathrm{M}$ ethanolamine, $\mathrm{pH}$ 8.0. Beads were then incubated for two hours at R/T in 10 volumes of ethanolamine. The beads were pelleted, supernatant aspirated, washed once in PBS $0.1 \% \mathrm{NaN}_{3}$ and stored at $4{ }^{0} \mathrm{C}$. 
Aliquots of 50\% (v/v) PAS slurry were removed before and after the DMP step. An equal volume of the sample buffer (5\% of $20 \%$ SDS [Severn Biotech Ltd., Worcestershire, UK], $5 \mathrm{mM}$ EDTA, $20 \mathrm{mM}$ Tris, $10 \%$ glycerol, $0.1 \mathrm{mg} / \mathrm{ml}$ bromophenol blue, $0.1 \mathrm{mg} / \mathrm{ml}$ pyronin $\mathrm{Y}$, $2 \mathrm{mM}$ phenylmethylsulfonyl fluoride [PMSF], $1 \%$ complete, $\mathrm{pH}$ 8.0) was added. Samples were incubated at $95^{\circ} \mathrm{C}$ for two minutes, centrifuged at $350 \mathrm{~g}$ for minutes at $\mathrm{R} / \mathrm{T}$, removed from the beads and stored at $-80^{\circ} \mathrm{C}$.

The OD of supernatant removed post PAS absorption was performed at OD280nm on M550 Double Beam Scanning UV-Visible spectrophotometer (Camspec, Leeds, UK) against the relevant buffer blank (PBS or high salt buffer). The remaining antibodies was calculated by using the formula: $\frac{O D 280}{A b \operatorname{constant}(=1.36)} \times$ volume of $A b$ in $\mu 1$.

Checking the efficiency of antibody crosslinking. The samples taken pre- and post-coupling were separated on $12.5 \%$ SDS-polyacrylamide gel under non-reducing conditions (as described later in the SDS-PAGE and blotting section), together with Mwt markers (SDS-7). Then the gels were stained in a solution of $0.1 \%$ Coomassie Blue $(50 \%(\mathrm{v} / \mathrm{v}) \mathrm{MeOH}, 5 \%(\mathrm{v} / \mathrm{v})$ glacial acetic acid) for 30 minutes with mixing, then de-stained with $0.06 \% \mathrm{MeOH}, 0.04 \%$ glacial acetic acid. Images were recorded by HP Scanjet 8300 (HP, California, US).

Preclearing the columns. The prepared columns were centrifuged for five minutes at $196 \mathrm{~g}$ and $4^{\circ} \mathrm{C}$ and supernatants were removed. Beads were washed twice with 0.1 glycine, $\mathrm{pH} 2$ to remove fragment light chains that were not coupled to heavy chains, as they may interfere with the results of the western blot analysis. After the preclearing, the columns were washed twice with PBS to return the $\mathrm{pH}$ to 7.4 before being stored at $4^{0} \mathrm{C}$.

\section{Preparation of erythrocyte membranes}

The method of preparing RBC membranes was adapted from Dodge et al. [16]. All equipment was chilled, and reagents were kept on ice during the preparation. The two EDTAblood samples (National Health Service Blood and Transplant, Bristol, UK) were centrifuged for five minutes at 3500rpm and supernatants were aspirated. The packed cells were washed thrice with PBS/100mM phenylmethylsulfonyl fluoride (PMSF) with $1 \%$ complete protease inhibitor cocktail with ethylenediaminetetraacetic acid (EDTA) (Complete Roche, Mannheim, Germany). Then $5 \mathrm{mM} \mathrm{NaP} / 100 \mathrm{mM}$ PMSF was added, and the samples were kept on ice for two minutes. Cells were centrifuged at $35,000 \mathrm{~g}$ for 10 minutes at $4^{\circ} \mathrm{C}$, the supernatant was aspirated, and the membranes were stored at $-80^{\circ} \mathrm{C}$.

\section{Proteins purification from solubilized membranes on antibody cross-linked columns}

Membranes were thawed at $4{ }^{\circ} \mathrm{C}$ then mixed with three volumes of solubilisation buffer (SB) (PBS containing 2\% [w/v] Triton X-100, 2.5mM EDTA, 2mM PMSF, 1:100 complete) for 30 minutes and then centrifuged for 15 minutes at $50,000 \mathrm{~g}$ and $4{ }^{\circ} \mathrm{C}$, to remove insoluble cytoskeletal proteins. Supernatants were incubated with $500 \mu$ PAS (pre-washed in SB) for one hour and then centrifuged for five minutes at $196 \mathrm{~g}$ and $4^{\circ} \mathrm{C}$. Supernatants were incubated 
with antibody cross-linked PAS for 15 hours at $4{ }^{\circ} \mathrm{C}$, and supernatant were harvested for successive incubation with other cross-linked PAS.

Proteins elution. After each incubation with the membrane lysates, cross-linked PAS were pelleted, washed thrice as above in SB, then eluted into 6-8 successive aliquots of $500 \mu 1$ of $0.1 \mathrm{M}$ glycine, $0.5 \% \mathrm{CHAPS}, 2 \mathrm{mM}$ PMSF, $2 \mathrm{mM}$ complete, $\mathrm{pH} 3.0$. The $\mathrm{pH}$ was corrected to $\mathrm{pH} 7.4$ by the addition of $25 \mu \mathrm{l}$ aliquots of $0.5 \mathrm{Mtris}$. A $10 \mu \mathrm{l}$ aliquot of each eluate fraction was solubilised in an equal volume of a non-reduced sample buffer. Each column was used to extract protein from the two RBC membrane lots. All aliquots of eluted proteins from each cross-linked column were pooled and concentrated by centrifuge on Centricon. For controlling the proteins purification, a $10 \mu 1$ sample was taken from each protein fraction, mixed with an equal volume of the SDS sample buffer with 4\% (v/v) 2-mercaptoethanol. Then the samples were analysed as described in the SDDS-PAGE and blotting section.

\section{Treatment of proteins with endoglycosidases}

\section{Endoglycosidases}

The following enzymes were used either alone or in combination: EndoH (Calbiochem, Germany), Endo $\beta$ and EndoF1. The digestion of EndoH, EndoF1 and Endo $\beta$ was in a pH6.1 buffer (2mM Na2HPO4.2H2O, 100mM Na2EDTA and 2\% 2-mercaptoethanol corrected with 2mM NaH2PO4.H2O, 100mM Na2EDTA and 2\% 2-mercaptoethano). PNGaseF was also used and the digestion of the enzyme was in PBS at pH7.4.

\section{CD44, CD47 and CD147 digestion}

Each of the concentrated proteins were divided into 10 aliquots of $10 \mu 1$ and an equal volume pH6.1 buffer ( 8 aliquots) or pH7.4 buffer ( 2 aliquots) was added. Endoglycosidases were added to appropriate aliquots: Endo-H=10mU/aliquot; Endo- $\beta=83.3 \mathrm{mU} / \mathrm{aliquot}$; EndoF1=10mU/aliquot; PNGaseF=3 $\mu \mathrm{l} /$ aliquot. Proteins were incubated for 18 hours at $37^{\circ} \mathrm{C}$. Samples were solubilised in an appropriate volume of $\mathrm{X} 2$ concentrated sample buffer and stored at $-80^{\circ} \mathrm{C}$. Each glycoprotein was digested one time and the results were reported.

\section{SDS-PAGE and blotting}

Gels of $10 \%$ (reduced and non-reduced) polyacrylamide gels (acrylamide/bisacrylamide ratio 37.5:1) with 3\% stack gel (acrylamide/bisacrylamide ratio 19:1) were run on the samples. Molecular weight (Mwt) markers- Novex ${ }^{\circledR}$ Sharp Pre-Stained Protein Standard and Magic Make XP western standard (Life technology, Carlsbad, USA) (mix), RBC membranes and proteins were electrophoresed in the gels by using Bio-Rad Mini Protein Tetra System (Bio-Rad) of 20mA/gel for approximately 90 minutes. Gels were blotted with Immobilon-P polyvinylidene difluoride (PVDF) (Millipore, Massachusetts, US) membranes under semidry conditions at $60 \mathrm{~mA}$ per-minigel on Trans-blot SD Semi-dry Transfer Cell (Bio-Rad) for one hour using $10 \%$ Tris-glycine-SDS buffer (25mM Tris, 192mM glycine, $0.1 \%$ [20\% solution] 
SDS). Membranes were blocked with $5 \%$ bovine serum albumin (BSA) in PBS and $0.08 \%$ Tween-20 (PBS-T) for one hour minimum. Membranes were incubated overnight at $4{ }^{\circ} \mathrm{C}$ with primary mAbs or animal polyclonal antibody in the blocking reagent. Membranes were washed four times with PBS-T and then incubated with alkaline phosphatase-conjugated secondary Abs to human IgG, rabbit IgG or mouse IgG1 (Jackson) for one hour at R/T, and then washed as before. Finally, the bands were visualised using Western Lightning CDP-Star Chemiluminescence reagent (Perkin Elmer, Waltham, USA) as per manufacturer instructions. Images were recorded on a Kodak Image Station 4000R using Kodak Imaging software.

\section{Results}

The glycosylated proteins CD44, CD147 and CD47 were isolated from solubilised RBC membranes by adsorbing and eluting from high-affinity antibody coupled to PAS (BRIC235, MA103 and BRIC126, respectively). The purified proteins then treated with different endoglycosidases.

\section{Ab-coupled PAS}

Quantity of Abs coupled to PAS beads.

The volumes of different Abs cross-linked to PAS were measured by spectrophotometry and the results were used to calculate the coupling efficiency. The results show a high coupling efficiency; the minimum percentage of Abs coupled to beads was 95\% (see Table 1).

Table 1. Spectrophotometry reading of samples and the coupling efficiency.

\begin{tabular}{|l|c|c|l|c|}
\hline $\begin{array}{c}\text { Abs samples taken } \\
\text { post-coupling }\end{array}$ & $\begin{array}{c}\text { OD } \\
(280)\end{array}$ & $\begin{array}{c}\text { Total } \\
\mathrm{mAb}\end{array}$ & $\begin{array}{c}\text { Amount of antibodies } \\
\text { uncoupled* }\end{array}$ & $\begin{array}{l}\text { Couplin } \\
\mathrm{g} \\
\text { efficiency }\end{array}$ \\
\hline BRIC235 & 0.064 & $2 \mathrm{mg}$ & $48 \mu \mathrm{g}$ & $98 \%$ \\
\hline BRIC126 & 0.142 & $2 \mathrm{mg}$ & $104 \mu \mathrm{g}$ & $95 \%$ \\
\hline MA103 & 0.034 & $3 \mathrm{mg}$ & $3 \mu \mathrm{g}$ & $99 \%$ \\
\hline
\end{tabular}

*the amount of uncoupled antibodies measured by the formula:

$$
\frac{O D 280}{A b \text { constant }(=1.36)} \times \text { volume of abtibodies }
$$

\section{Efficiency of coupling antibody to beads.}

Samples taken before and after cross-linking Abs to PAS beads were run in $12.5 \%$ SDSPAGE to examine the success of coupling Abs to PAS. The results clearly show the effectiveness of coupling. Both light and heavy chains were eluted before coupling (see 
Figure 1A). However, as expected, after cross-linking to PAS with DMP, only small quantity of light chains were present in the gel and bands of heavy chains apparently disappear, indicating that the vast majority of the Abs were coupled (see Figure 1B).

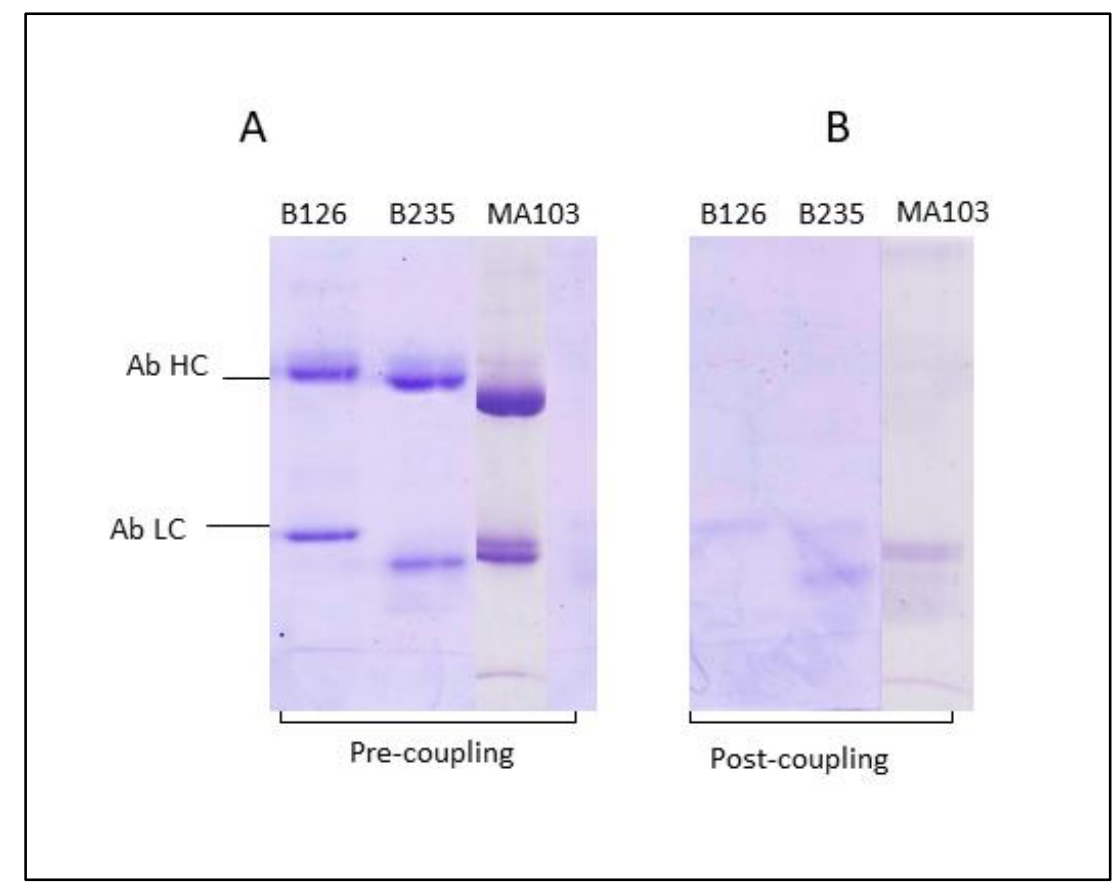

Figure '. The effectiveness of cross-linked antibodies to PAS. (A) Running of samples taken before coupling Abs to PAS. (B) Running of samples taken after coupling Abs to PAS. B126, BRIC126 Ab; B235, BRIC235 Ab; Ab HC, Antibody haevy chains; Ab LC, Antibody light chains. 12.5\% SDS-PAGE gel stained with Coomassie Blue.

\section{Protein purification from membranes and deglycosylated treatment}

Solubilised material from $3 \mathrm{ml}$ membranes from two donors was incubated with each $\mathrm{mAb}$ column and eluted. Aliquots from each of the eluted fractions were analysed by SDS-PAGE and immunoblotting with relevant mAbs before pooled and concentrated and again analysed by SDS-PAGE. The concentrated proteins then treated with different endoglycosidases and investigated as seen below.

Fractions of protein elutions were pooled and concentrated. The CD147 and CD47 elutions were pooled and concentrated to $150 \mu \mathrm{l}$, as they were estimated to have a high concentration of proteins while CD44 was concentrated to $125 \mu$ l.

\section{CD44}

When CD44 fractions were pooled and concentrated, samples revealed to have a high quantity of standard CD44 after blotted with the relevant mAbs (see Figure 2) although the fractions eluted from the CD44 column were shown to have a low concentration of the protein 
(data not shown). As shown, KZ1 and BRIC 222 bind to the $80 \mathrm{kDa}$ erythrocyte form of CD44.

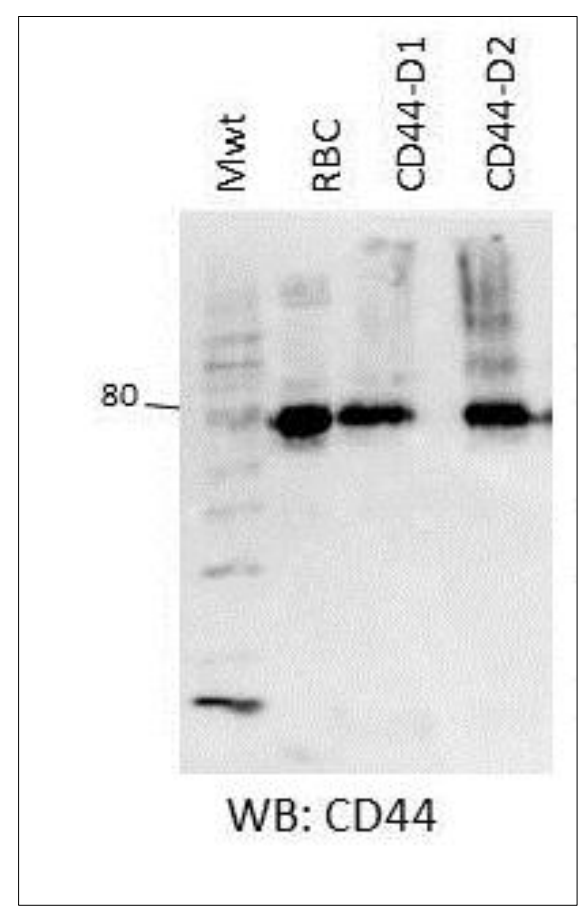

Figure $r$. Presence of CD44 in the concentrated extraction. CD44 purified from RBC membranes were probed with anti-CD44 (KZ1 and BRIC222). Protein was loaded in $10 \%$ reducing SDS. RBC, RBC membranes; CD44-D1, CD44 purified from Donor 1; CD44-D2, CD44 purified from Donor 2; WB, specificity of antibody used to probe the immunoblot.

The purified CD44 was deglycosylated with different enzymes: EndoH, Endo $\beta$, EndoF1 and PNGaseF as seen in Figure 3. The effect of deglycosylation was examined by blotting the treated protein with anti-CD44 (KZ1) after separated on 10\% SDS-PAGE. PNGase F showed to have the strongest effects on the glycoprotein where most CD44s were partially deglycosylated and the band shifted to $60 \mathrm{kDa}$ suggesting that the cores of $\mathrm{N}$-linked oligosaccharide chains are not fucosylated. Some CD44 N-glycans were susceptible for EndoH but apparently not Endo $\beta$ or EndoF1. Combination of them did not reveal stronger effects. 


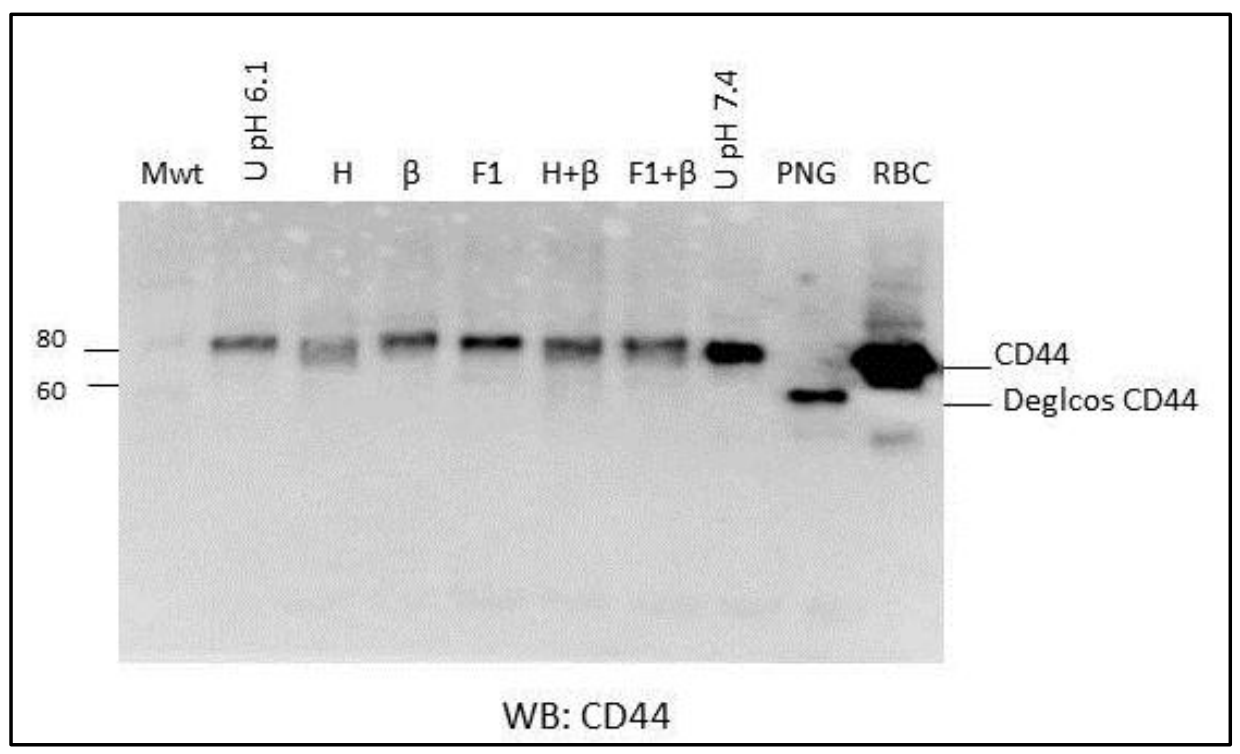

Figure $r$. Effectiveness of enzyme deglycosylation in CD44. Anti-CD44 (KZ1) reactivity with enzymes treated CD44. Protein was loaded in 10\% non-reducing SDS. U pH6.1, untreated CD44 in pH6.1 buffer; H, CD treated with EndoH; $\beta$, Endoß; F1, EndoF1; U pH7.4, untreated sample in pH7.4; PNG, PNGaseF; WB, specificity of antibody used to probe the immunoblot.

\section{CD47}

Elution fractions of CD47 extracted from solubilised RBC membranes by using the BRIC126 column were blotted with the relevant mAb (see Figure 4A) before concentrated and then probed with CD47 polyclonal antibody (Out-1) (see Figure 4B). The CD47 molecule reveal broad band extending from $\sim 45 \mathrm{kDa}$ to $65 \mathrm{kDa}$ indicating its highly heterogeneity.

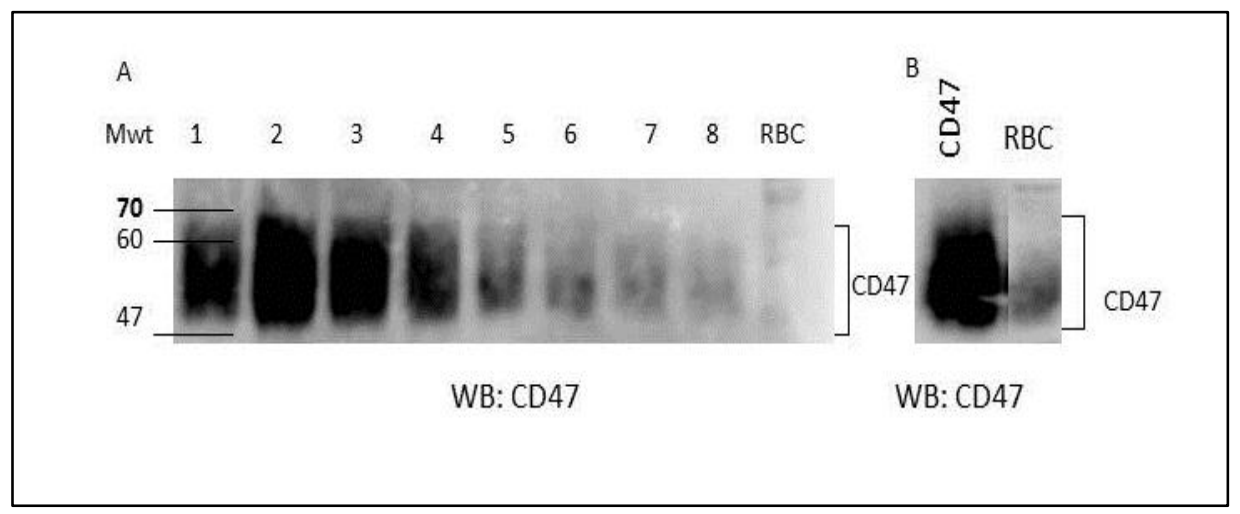

Figure \& . Purified CD47. (A) Presence of CD47 in the CD47 column fractions (B) after concentrating the fractions. Purified CD47 (5 $\mu$ g protein) probed with CD47 polyclonal antibody (Out-1). The samples were separated in (A) $10 \%$ reducing (B) non-reducing SDS-PAGE. 1, 2, 3, 4, 5, 6, 7 and 8, fraction 1, 2, 3, 4, 5, 6, 7 and 8, respectively; RBC, RBC membranes; WB, specificity of antibody used to probe the immunoblot. 
Immunoblotting the enzyme-treated CD47 with relevant Abs demonstrated that the linked $\mathrm{N}$-glycans were remarkably susceptible for PNGaseF. As seen in Figure 5, EndoH and EndoF1 causing only partial digestion indicating that the large proportion of CD47 N-linked oligosaccharide chains are from complex type as this type resist to both enzymes. Similarly to $\mathrm{CD} 44$, there was no noticeable effect of Endo $\beta$ alone, and when it combined with EndoH or EndoF1 no digestion enhancement was detected. The higher molecular mass material was most likely due to aggregation of the protein fragments suggesting that the molecule tend to aggregate at lower $\mathrm{pH}$ in comparing with natural $\mathrm{pH}$.

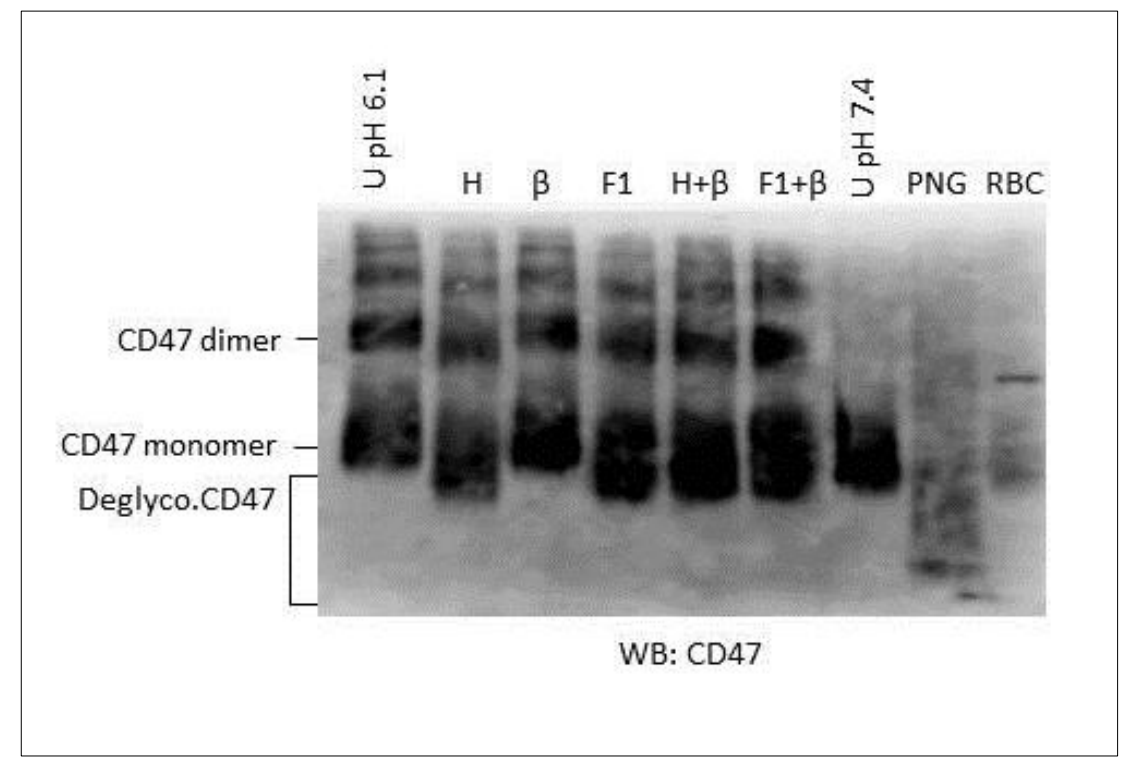

Figure $^{\circ}$. Effectiveness of enzyme deglycosylation in CD47. Polyclonal anti-CD147 (Out1) reactivity with enzyme-treated CD47. U pH6.1, untreated CD47 in pH6.1 buffer; $H$, CD47 treated with EndoH; $\beta$, Endoß, F1, EndoF1; U pH7.4, untreated sample in pH7.4; PNG, PNGaseF; WB, specificity of antibody used to probe the immunoblot.

\section{$C D 147$}

As CD47, elutions from CD147 column show high concentration of the purified protein (Figure 6). The highly glycosylated purified CD147 revealed broad band extend from around $40 \mathrm{kDa}$ to $80 \mathrm{kDa}$ (Figure 6A), and when analyse higher quantity the band defused as seen in Figure $6 \mathrm{~B}$ revealing the heterogeneous N-glycosylation. 


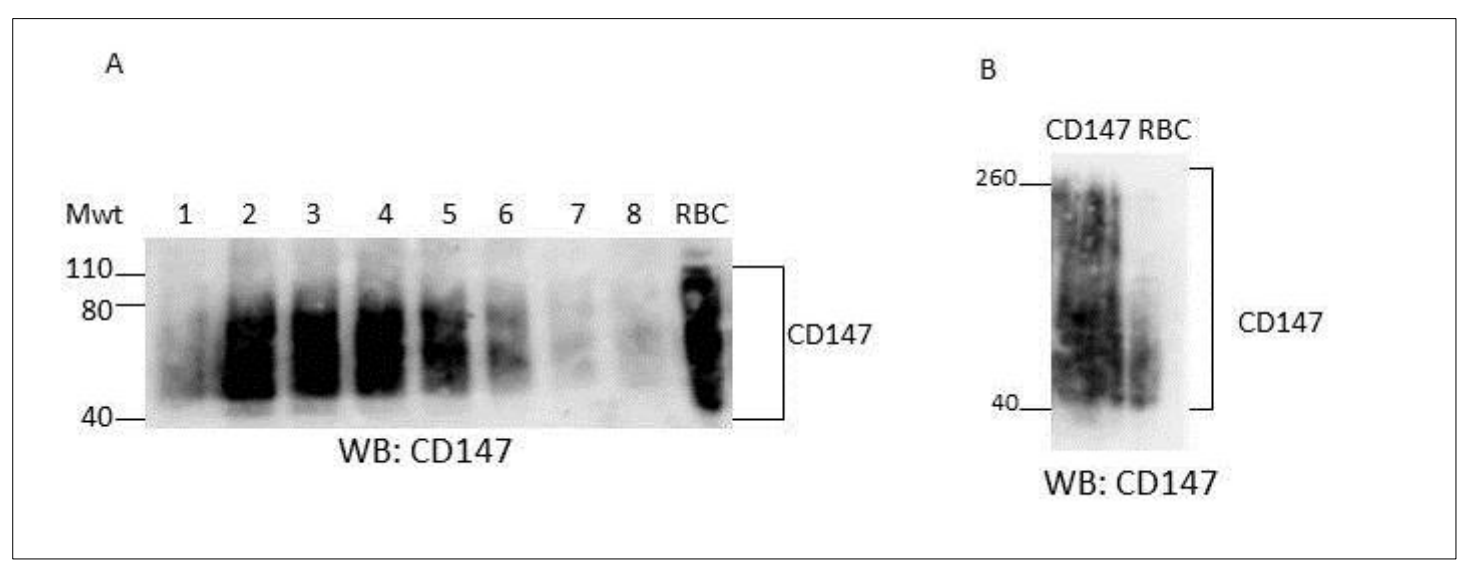

Figure 7 . Purified CD147. (A) Presence of CD147 in the CD147 column fractions (B) after concentrating the fractions. Purified CD147 (5 and 7.5 $\mu \mathrm{g}$ protein) probed with CD147 biotinylated Ab. The samples were separated in (A) 10\% reducing (B) nonreducing SDS-PAGE. 1, 2, 3, 4, 5, 6, 7 and 8 , fraction $1,2,3,4,5,6,7$ and 8 , respectively; RBC, RBC membranes; WB, specificity of antibody used to probe the immunoblot.

Variability in the mobility of the enzymes treated purified CD147 was illustrated in Figure 7. Interestingly, after treated the samples with EndoH no bands were detected when blotting with the anti-CD147. PNGaseF effectively deglycosylated CD147, since a remarkable Mwt shifting of the band was present. Comparing with the control, there was a slightly decrease in band condensation of the protein treated with Endo $\beta$ indicating that the enzyme cleaved only a small proportion of the $\mathrm{N}$-glycans. However, the protein $\mathrm{N}$-glycosyl-linked oligosaccharide chains apparently resist to EndoF1 as there was not a detectable change in the molecular mass.

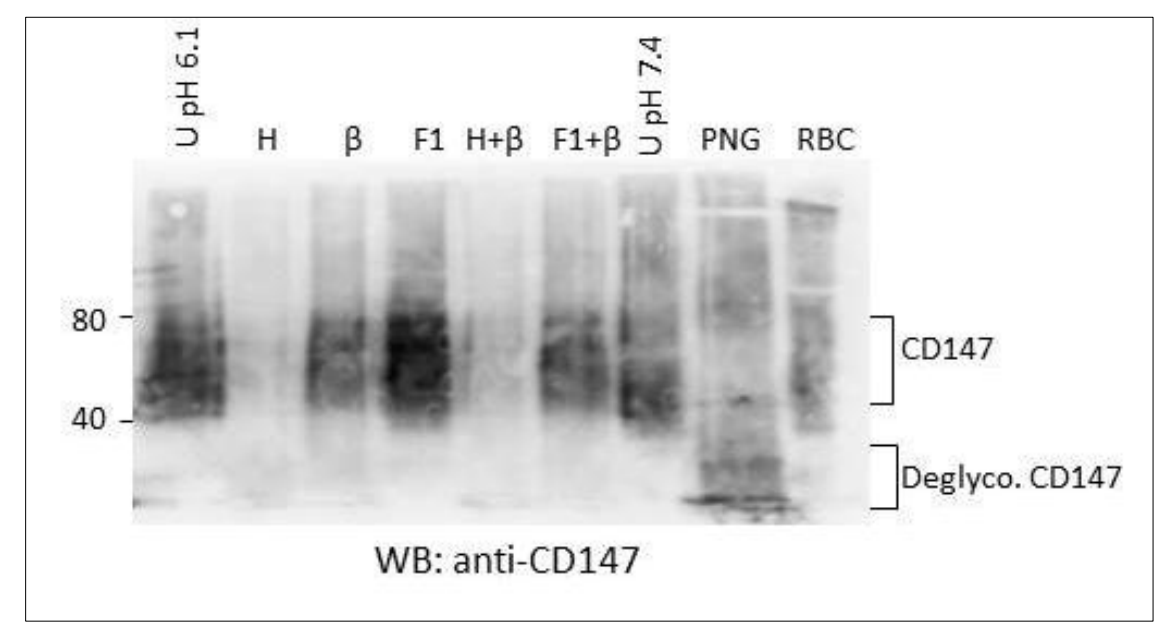

Figure $\checkmark$. Effectiveness of enzyme deglycosylation in CD147. Polyclonal anti-CD147 (biotinylated) reactivity with enzymes treated. U pH6.1, untreated CD147 in pH6.1 buffer; H, CD147 treated with EndoH; $\beta$, Endoß, F1, EndoF1; U pH7.4, untreated sample in pH7.4; PNG, PNGaseF; WB, specificity of antibody used to probe the immunoblot. 


\section{Discussion}

In our study we isolated three glycoproteins, CD44, CD47 and CD147, from RBC membranes using antibody high-affinity chromatography. Effects of the endoglycosidases PNGaseF, EndoF1, EndoH and Endo $\beta$ on these glycoproteins were examined. Knowing the effects of endoglycosidases on glycoproteins provides clues about the structure of their Nlinked glycans.

CD44 cell surface glycoprotein is present on a broad range of cells and tissues. The CD44 family is heterogeneous, and a number of isoforms are expressed on different cells, referring to alternative splicing in the controlled gene. It has been suggested that the Mwt of CD44 before post-translation modification is $37 \mathrm{kDa}$ [17]. Our findings show that the Mwt of erythrocyte CD44 is $80 \mathrm{kDa}$, which is consistent with the results of other studies $[18,19]$. It is the widely expressed isoform of CD44 that does not contain the product of differentially spliced exons [20]. Part of the variation in the $\sim 40 \mathrm{kDa}$ is attributed to extensive glycosylation of the molecule. Different cells expressing this molecule have different glycosylation forms [21]. There are five expected $\mathrm{N}$-glycosylation sites and a number of O-linked glycosylation sites in the extracellular region of the molecule. Deglycosylating the purified CD44 shows that some of the N-glycans are susceptible to EndoH but not EndoF1. Both enzymes have the ability to cleave high-mannose and hybrid, but not complex, oligosaccharide chains at the bond between the two innermost GlcNac groups. However, the ability of EndoF1 to cleave Nglycans is significantly reduced by the presence of a fucosylated core, while this does not change the effectiveness of EndoH [22]. Thus, this result indicates the presence of some fucosylated $\mathrm{N}$-glycan cores with $\alpha 1-6$ fucose residue bound to the innermost GlcNAc residue. The findings also suggest that there are no significant poly- $\mathrm{N}$-acetyllactosamine groups on the CD44 N-glycans, as no noticeable effects were detected on the Mwt after treatment with Endo $\beta$. This is in line with the findings of Spring et al. [18].

PNGaseF removes a considerable amount of N-glycans, causing remarkable shifting in the treated molecule's SDS-PAGE band, which is consistent with another study [23]. The findings suggest that a significant number of the $\mathrm{N}$-linked oligosaccharides were complex types, because they were susceptible to PNGaseF although they were not cleaved effectively by the other enzymes. However, some oligosaccharides were not affected, suggesting they may be from O-linked glycan and/or PNGaseF-resistant oligosaccharides such as the uncommon form of N-glycans with $\alpha$ 1-3 Fuc on the GlcNac bound to Asn [24].

CD47 is expressed widely on human cells, including most hematopoietic cells [25]. The results show a diffuse band $(47-70 \mathrm{kDa})$ of the purified CD47, indicating the heterogeneity of the attached oligosaccharides. This is similar to results obtained by other studies [26,27]. Immunoprecipitation of CD47 from intact erythrocytes shows a narrower band (47-52 kDa) [28]. Purifying the molecules from other cells revealed a $\sim 50-\mathrm{kDa}$ band [27]. We found that purified CD47 molecules at low $\mathrm{pH}$ form two segregated bands at higher Mwt, which are expected to be aggregations of dimers and tetramers. Brown et al. [27] suggested that samples incubated at high temperature $\left(100^{\circ} \mathrm{C}\right)$ can cause aggregation of the glycoprotein molecules 
run on SDS-PAGE. Also, CD47 from intact erythrocytes revealed a distinct band at high molecular mass [28]. The molecule is heavily glycosylated and carries six potential N-glycan sites, most located near its N-terminus [29]. Results from treating CD47 with different endoglycosidases suggest that only some of the linked N-glycans are of hybrid type with a non-fucosylated core, as there was a slight reduction in the Mwt when treated with EndoH and EndoF1. Avent et al. [28] found that N-glycans were apparently not susceptible to digestion by Endo $\beta$, suggesting that they do not contain extensive poly-N-acetyllactosaminyl groups. By contrast, PNGaseF, which can cleave complex and branched structures, efficiently deglycosylated CD47. This result is consistent with the findings of Mawby et al. [30], who also detected the 26-kDa deglycosalted CD47 band.

CD147 expresses widely on different cell types. However, although these cells apparently have very similar CD147 polypeptides with $\sim 27-\mathrm{kDa}$ molecular mass [31-33], some show different glycosylation and cell-type-dependent glycosylation [33,34]. Our study detected a broad, diffuse band of the erythrocyte CD147, which is inconsistent with the Mwt of the molecule from RBC [35] and other cell types [36] (which extends from $\sim 35$ to $\sim 70 \mathrm{kDa}$ ). The high heterogeneity of oligosaccharide chains causes the characteristic diffuse band of CD147. Around half of its Mwt is referred to the linked carbohydrates [33]. There is little information in the literature related to glycosylation of erythrocyte CD147. It has been suggested that CD147 N-linked glycans contain a significant amount of poly-N-acetyllactosamine structures $[31,36]$. However, our results on erythrocytes disagree, as samples treated with endo $\beta$ show only a slight variation in the intensity of the band and no detectable shift in the Mwt. This may have resulted from removing short repeating units of poly- $\mathrm{N}$-acetyllactosamine present in the three N-linked glycosylation sites on the molecule, where these structures might be the antigen determinant of the anti-CD147 used. However, the presence of branched polylactosamine units can significantly block endo $\beta$ digestion [8]. By contrast, most CD147 was effectively deglycosylated by PNGaseF and EndoH. This was also observed with highly and less glycosylated forms of CD147 purified from different cells [31]. Like CD44, CD147 was digested by EndoH but not EndoF1, indicating that the N-glycans are high-mannose and/or hybrid, and fucosylated. The deglycosylated molecules were not detected by SDSPAGE, as either they may fall at the end of the gel, or the primary antibody epitopes were affected. As there are glycosylation variations identified in some glycosylations associated with malignant transformation [37] and roles of CD147 in tumour progression [38], it would be worth investigating possible variation in CD147 glycosylation of glycoproteins of normal and abnormal erythrocytes.

\section{Conclusion}

Extracting CD47, CD44 and CD147 using BRIC126, BRIC235 and MA103 columns, respectively, was successfully achieved. This study illustrates the effects of different endoglycosidases on purified glycoproteins, combining this information in a single analysis. We found that PNGaseF effectively deglycosylated the examined glycoproteins. Interestingly, EndoH was also able to deglycosylate the molecules, albeit to a different degree. EndoF1 showed some effectiveness in removing some $\mathrm{N}$-linked glycans carried by CD47. The 
absence of any observed effect of Endo $\beta$ on CD44 and CD47 may result from either resistance to degradation of an $\mathrm{N}$-glycosyl-linked oligosaccharide or the lack of polylactosamine segments. The effects of various enzymes on the glycoproteins give clues regarding the possible biochemical structure of their $\mathrm{N}$-glycans.

\section{Declaration}

The author declares no competing financial interests.

\section{Acknowledgment}

The research supported by National Institute for Health Research. I thank Dr Frances Spring (National Health Service Blood and Transplant) who provided insight, expertise and unlimited support to the research. Also thank King Saud University for providing me an opportunity to do the research in NHSBT Blood Centre Filton, Bristol, United Kingdom.

\section{References}

Parodi, A. J. Role of N-Oligosaccharide Endoplasmic Reticulum Processing Reactions in Glycoprotein Folding and Degradation. Biochem. J. 2000, 348 Pt 1, 1-13..

Wormald, M. R.; Dwek, R. A. Glycoproteins: Glycan Presentation and Protein-Fold Stability. Structure 1999, 7, R155-R160.

Denecke, J. Congenital Disorders of Glycosylation: Review of Their Molecular Bases, Clinical Presentations and Specific Therapies. 2003. DOI:10.1007/s00431-002-1136-0

Apweiler, R.; Hermjakob, H.; Sharon, N. On the Frequency of Protein Glycosylation, as Deduced from Analysis of the SWISS-PROT Database. Biochim. Biophys. Acta 1999, 1473, $4-8$.

Denecke, J. et al. Characterization of the N-Glycosylation Phenotype of Erythrocyte Membrane Proteins in Congenital Dyserythropoietic Anemia type II (CDA II/HEMPAS). Glycoconj. J. 2008, 25, 375-382.

Freeze, H. H.; Kranz, C. Endoglycosidase and Glycoamidase Release of N-Linked Glycans. Curr. Protoc. Mol. Biol. 2010, Chapter 17, Unit 17.13A.

Gattegno, L.; Bladier, D.; Garnier, M.; Cornillot, P. Changes in Carbohydrate Content of Surface Membranes of Human Erythrocytes during Ageing. Carbohydr. Res. 1976, 52, 197208.

Plummer, T. H.; Elder, J. H.; Alexander, S.; Phelan, A. W.; Tarentino, A. L. Demonstration of Peptide:N-Glycosidase F Activity in Endo- $\beta$-N-Acetylglucosaminidase F Preparations. $J$. Biol. Chem. 1984, 259, 10700-10704. 
Schenkel-Brunner, H. Human Blood Groups: Chemical and Biochemical Basis of Antigen Specificity. Springer-Verlag Wien GmbH, 2000. DOI:10.1007/978-3-7091-6294-1

Scudder, P.; Uemura, K.; Dolby, J.; Fukuda, M. N.; Feizi, T. Isolation and Characterization of an Endo-Beta-Galactosidase from Bacteroides fragilis. Biochem. J. 1983, 213, 485-494.

Mallinson, G. et al. Identification and Partial Characterization of the Human Erythrocyte Membrane Component(s) that Express the Antigens of the LW Blood-Group System.

Biochem. J 1986, 234, 649-652.

Tanner, M. J. A.; Anstee, D. J.; Mallinson, G.; Ridgwell, K.; Martin, P. G.; Avent, N. D.; Parsons, S. F. Effect of Endoglycosidase F-Peptidyl N-Glycosidase F Preparations on the Surface Components of the Human Erythrocyte. Carbohydr. Res. 1988, 178 (1), 203-212.

Kwong, F. Y. P. et al. Erythrocyte Nucleoside and Sugar Transport Endo-/I-Galactosidase and Endoglycosidase-F Digestion of Partially Purified Human and Pig Transporter Proteins. Biochem. J 1986, 240, 349-356.

Lienhard, G. E.; Crabb, J. H.; Ransome, K. J. Endoglycosidase F Cleaves the Oligosaccharides from the Glucose Transporter of the Human Erythrocyte. Biochim. Biophys. Acta-Biomembr. 1984, 769, 404-410.

Moser, J. J.; Chan, E. K. L.; Fritzler, M. J. Optimization of Immunoprecipitation-Western Blot Analysis in Detecting GW182-Associated Components of GW/P Bodies. Nat. Protoc. 2009, 4, 674-685.

Dodge, J. T.; Mitchell, C.; Hanahan, D. J. The Preparation and Chemical Characteristics of Hemoglobin-Free Ghosts of Human Erythrocytes. Arch. Biochem. Biophys. 1963, 100 , 119130.

Goldstein, L. A.; Zhou, D. H.; Picker, L.; Minty, C.; Bargatze, R.; Ding, J,; Butcher, E. A Human Lymphocyte Homing Receptor, the Hermes Antigen, Is Related to Cartilage Proteoglycan Core and Link Proteins. Cell 1989, 56, 1063-1072 .

Spring, F. et al. The Ina and Inb Blood Group Antigens Are Located on a Glycoprotein of 80,000 MW (the CDw44 Glycoprotein) Whose Expression Is Influenced by the In(Lu) Gene. Immunology 1988, 64, 37-43..

Anstee, D. J.; Gardner, T. B.; Springt, F. A.; Holmes, C. H.; Simpson, K. L.; Parsons, S. F.; Mallinson, G.; Yousaf, S. M.; Judsonj, P. A. New Monoclonal Antibodies in CD44 and CD58: Their Use to Quantify CD44 and CD58 on Normal Human Erythrocytes and to Compare the Distribution of CD44 and CD58 in Human Tissues. Immunology 1991, 74, 197205.

Goodison, S.; Urquidi, V.; Tarin, D. CD44 Cell Adhesion Molecules. J Clin Pathol Mol Pathol. 1999, 52, 189-196. 
Brown, T. A.; Bouchard, T.; St. John, T.; Wayner, E.; Carter, W. G. Human Keratinocytes Express a New CD44 Core Protein (CD44E) as a Heparan-Sulfate Intrinsic Membrane Proteoglycan with Additional Exons. J. Cell Biol. 1991, 113, 207-221.

Tarentino, A. L.; Plummer, T. H. Enzymatic Deglycosylation of Asparagine-Linked Glycans: Purification, Properties, and Specificity of Oligosaccharide-Cleaving Enzymes from Flavobacterium meningosepticum. Methods Enzymol. 1994, 230, 44-57..

Delcommenne, M.; Kannagi, R.; Johnson, P. TNF- $\alpha$ Increases the Carbohydrate Sulfation of CD44: Induction of 6-Sulfo N-Acetyl Lactosamine on N- and O-Linked Glycans. Glycobiology 2002, 12, 613-622.

Tretter, V.; Altmann, F.; Marz, L. Peptide-N4-(N-Acetyl-/3-Glucosaminyl)Asparagine Amidase F Cannot Release Glycans with Fucose Attached a1 + 3 to the Asparagine-Linked N-Acetylglucosamine Residue. Eur. J. Biochem 1991, 299, 647-652.

Schmidt, R.; Feller, A.; Hadam, M.; Johnson, J. Schuberl, J.; Schwinzer, R.; Stoll, M.; Pp, W.; Rialto, P.; Sdm, H, et al. At the 4th International Workshop on Human Leucocyte Differentiation Anti-gens Towards a Better Definition d huron Leucoc e Surface Molecules. Immunology Today 1989, 10, 253-258.

Mawby, W. J.; Holmes, C. H.; Anstee, D. J.; Spring, F. A.; A Tanner, M. J. Isolation and Characterization of CD47 Glycoprotein: A Multispanning Membrane Protein which Is the Same as Integrin-Associated Protein (IAP) and the Ovarian Tumour Marker OA3. Biochem. J 1994, 304, 525-530.

Brown, E.; Hooper, L.; Ho, T.; Gresham, H. Integrin-Associated Protein: A 50-kD Plasma Membrane Antigen Physically and Functionally Associated with Integrins. J. Cell Biol. 1990, $111,2785-2794$.

Avent, N.; Judson, P. A.; Parsons, S. F.; Mallinson, G.; Anstee, D. J.; Tanner, M. J.; Evans, P. R.; Hodges, E.; Maciver, A. G.; Holmes, C. Monoclonal Antibodies That Recognize Different Membrane Proteins That Are Deficient in Rhnull Human Erythrocytes. One Group of Antibodies Reacts with a Variety of Cells and Tissues Whereas the Other Group Is Erythroid-Specific. Biochem J 1988, 251, 499-505.

Brown, E. J.; Frazier, W. A. Integrin-Associated Protein (CD47) and Its Ligands. Trends in Cell Biology 2001, 11, 130-135.

Mawby, W. J.; Holmes, C. H.; Anstee, D. J.; Spring, F. A.; A Tanner, M. J. Isolation and Characterization of CD47 Glycoprotein: A Multispanning Membrane Protein Which Is the Same as Integrin-Associated Protein (IAP) and the Ovarian Tumour Marker OA3. Biochem. J 1994, 304, 525-530.

Tang, W.; Chang, S. B.; H. Links between CD147 Function, Glycosylation, and Caveolin-1. Mol. Biol. Cell 2004, 16, 1-13. 
Miyauchi, T.; Kanekura, T.; Yamaoka, A.; Ozawa, M.; Miyazawa, S.; Muramatasu, T. Basigin, a New, Broadly Distributed Member of the Immunoglobulin Superfamily, Has Strong Homology with Both the Immunoglobulin V Domain and the $\beta$-Chain of Major Histocompatibility Complex Class II Antigen. J. Biochem. 1990, 107, 316-323.

Yu, X.-L.; Jiang, J.-L.; Li, L.; Feng, Q.; Xu, J.; Chen, Z.-N. The Glycosylation Characteristic of Hepatoma-Associated Antigen HAb18G/CD147 in Human Hepatoma Cells. Int. J. Biochem. Cell Biol. 2006, 38, 1939-1945.

Li, R.; Huang, L.; Guo, H.; Toole, B. P. Basigin (Murine EMMPRIN) Stimulates Matrix Metalloproteinase Production by Fibroblasts. J. Cell. Physiol. 2001, 186, 371-379.

Williams, B. P. et al. Biochemical and Genetic Analysis of the Ok a Blood Group Antigen. 1988, 27, 322-329.

Bai, Y.; Huang, W.; Ma, L.-T.; Jiang, J.-L.; Chen, Z.-N. Importance of N-Glycosylation on CD147 for Its Biological Functions. Int. J. Mol. Sci. 2014, 15, 6356-6377.

Bai, Y.; Huang, W.; Ma, L.-T.; Jiang, J.-L.; Chen, Z.-N. Importance of N-Glycosylation on CD147 for Its Biological Functions. Int. J. Mol. Sci. 2014, 15, 6356-6377.

Ya, L.; Zucker, S.; Toole, B. P. Roles of the Multifunctional Glycoprotein, Emmprin (Basigin; CD147), in Tumour Progression. ThrombHaemost 2005, 93, 199-204. 


\title{
The Effects of Different Endoglycosidases on Erythrocyte CD44, CD47 and CD147 Purified by Affinity Chromatography
}

\author{
مهرة عبدالعزيز محمد \\ معيدة بقسم المختبر ات الإكلينيكيةـ كلية العلوم الطبية التطبيقية- جامعة الملك سعود
}

\section{ملخص البحث}

الإندوجلايكوسايديز(endoglycosidase) هي عبارة عن أنزيمات لها القدرة على فصل أنواع العاع محددة

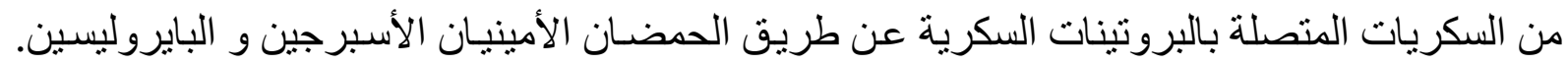

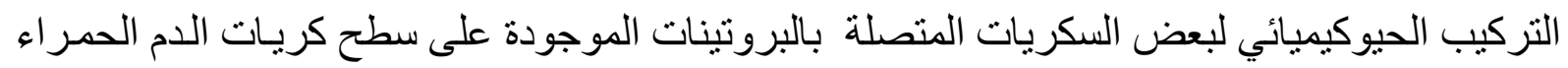

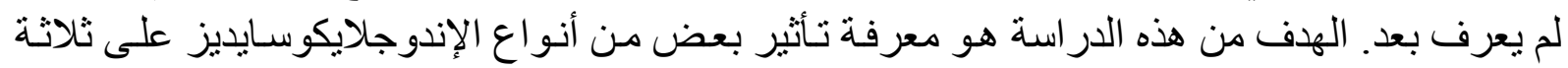

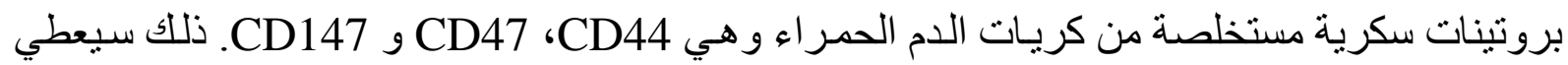

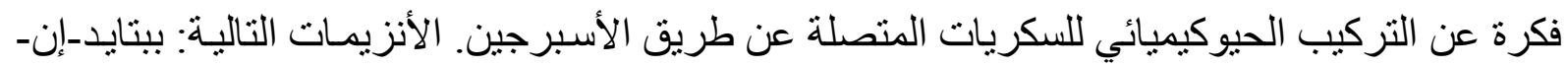

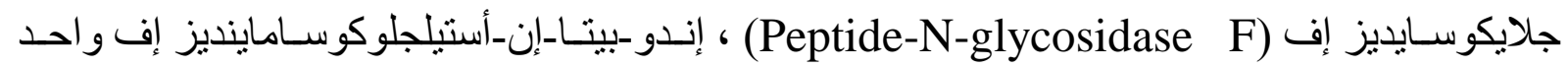
Endo-) (Endo- $\beta-N$-acetylglucosaminidase F1)

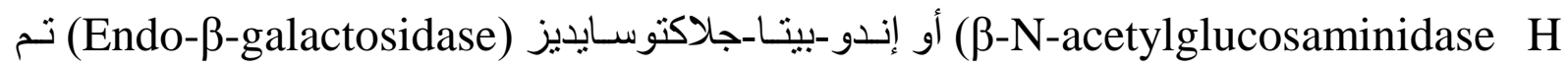

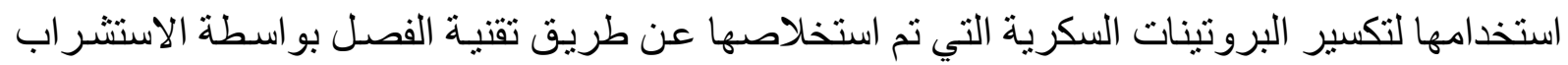

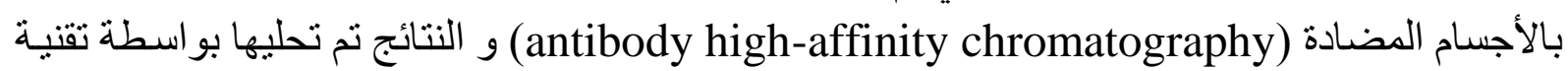
الفصل الكهربائي لهلام كبريتات دوديكل الصوديوم متعدد الأكريلامايد (podium dodecyl sulfate) CD44 polyacrylamide gel electrophoresis

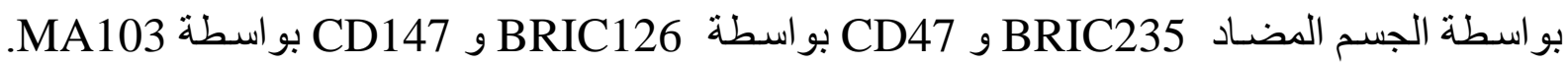

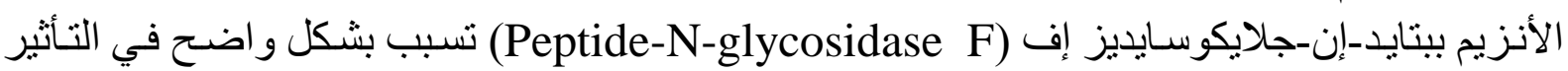
على سر عة تحرك البروتينات السكرية في الهلام بينما لم يلحظ تأثثر يذكر للأنزيم إندو -بيتا-جلاكتوسايديز

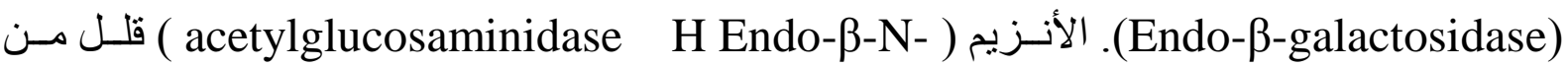

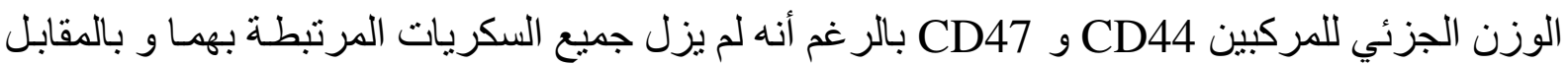

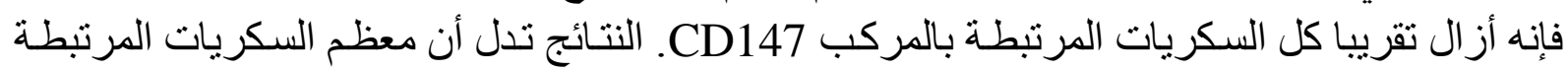

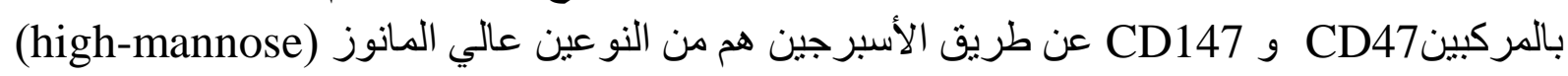

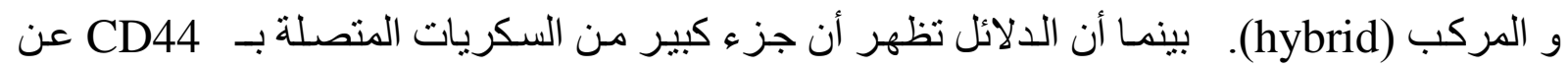
طريق الأسبرجين هي من النوع المعقد (complex). 